\title{
IUCrJ
}

Volume 3 (2016)

Supporting information for article:

Whole-pattern fitting technique in serial femtosecond nanocrystallography

Ruben A. Dilanian, Sophie R. Williams, Andrew V. Martin, Victor A. Stretsov and Harry M. Quiney 


\section{Whole-pattern fitting technique in serial femtosecond nanocrystallography}

Ruben A. Dilanian, Sophie R. Williams, Andrew V. Martin, Victor A. Stretsov and Harry M. Quiney

\section{Supplementary Information}

Table S1. Structure factor moduli of selected Bragg reflections extracted from a merged diffraction pattern from the test protein using the whole-pattern fitting approach, $\left|F^{W P F}\right|$, the integration approach, $\left|F^{I}\right|$, and calculated using published data (PDB ID: 4AK8), $\left|F^{C}\right| . \Omega^{W P F}$ and $\Omega^{I}$ are the relative errors for $\left|F^{W P F}\right|$ and $\left|F^{I}\right|$ respectively. The diffraction pattern was calculated in the $(h k 0)$ crystallographic plane from a set of 2000 crystallites with a mean of 30 unit-cells along [100] direction and of 10 unit-cells along the [010] direction. The diffraction pattern was simulated on a 340x340 pixel array to 5.0 A resolution. The average relative errors, $\left\langle\Omega^{W P F}\right\rangle=0.06$ and $\left\langle\Omega^{I}\right\rangle=0.13$, have been calculated with several weak reflections excluded (indicated in bold).

\begin{tabular}{|c|c|c|c|c|c|c|c|c|c|c|c|c|c|c|c|}
\hline$h$ & $k$ & $l$ & $\left|F^{C}\right|$ & $\left|F^{W P F}\right|$ & $\Omega^{W P F}$ & $\left|F^{\prime}\right|$ & $\Omega^{I}$ & $h$ & $k$ & $l$ & $\left|F^{C}\right|$ & $\left|F^{W P F}\right|$ & $\Omega^{W P F}$ & $\left|F^{I}\right|$ & $\Omega^{I}$ \\
\hline 15 & 15 & 0 & 1479.44 & 1387.84 & 0.06 & 1392.08 & 0.06 & 9 & 15 & 0 & 348.55 & 334.33 & 0.04 & 348.15 & 0.00 \\
\hline 15 & 14 & 0 & 765.45 & 721.29 & 0.06 & 673.07 & 0.12 & 9 & 14 & 0 & 1691.03 & 1580.70 & 0.07 & 1653.20 & 0.02 \\
\hline 15 & 13 & 0 & 719.08 & 667.83 & 0.07 & 719.89 & 0.00 & 9 & 13 & 0 & 601.14 & 559.13 & 0.07 & 618.65 & 0.03 \\
\hline 15 & 12 & 0 & 119.05 & 118.77 & 0.00 & 61.28 & 0.49 & 9 & 12 & 0 & 427.85 & 413.24 & 0.03 & 469.07 & 0.10 \\
\hline 15 & 11 & 0 & 341.29 & 321.21 & 0.06 & 297.45 & 0.13 & 9 & 11 & 0 & 718.64 & 673.14 & 0.06 & 739.90 & 0.03 \\
\hline 15 & 10 & 0 & 140.19 & 137.59 & 0.02 & 183.96 & 0.31 & 9 & 10 & 0 & 112.06 & 106.48 & 0.05 & 106.82 & 0.05 \\
\hline 15 & 9 & 0 & 175.08 & 171.20 & 0.02 & 147.19 & 0.16 & 9 & 9 & 0 & 164.33 & 152.49 & 0.07 & 184.38 & 0.12 \\
\hline 15 & 8 & 0 & 165.56 & 164.25 & 0.01 & 152.63 & 0.08 & 9 & 8 & 0 & 552.65 & 521.81 & 0.06 & 458.84 & 0.17 \\
\hline 15 & 7 & 0 & 1122.08 & 1033.00 & 0.08 & 1219.34 & 0.09 & 9 & 7 & 0 & 746.68 & 704.97 & 0.06 & 766.41 & 0.03 \\
\hline 15 & 6 & 0 & 885.83 & 833.21 & 0.06 & 925.19 & 0.04 & 9 & 6 & 0 & 153.23 & 148.84 & 0.03 & 121.82 & 0.20 \\
\hline 15 & 5 & 0 & 564.17 & 536.45 & 0.05 & 473.25 & 0.16 & 9 & 5 & 0 & 276.23 & 259.15 & 0.06 & 284.48 & 0.03 \\
\hline
\end{tabular}




\begin{tabular}{|c|c|c|c|c|c|c|c|c|c|c|c|c|c|c|c|}
\hline 15 & 4 & 0 & 71.54 & 63.84 & 0.11 & 87.91 & 0.23 & 9 & 4 & 0 & 117.62 & 108.81 & 0.07 & 121.12 & 0.03 \\
\hline 14 & 15 & 0 & 167.96 & 170.06 & 0.01 & 118.12 & 0.30 & 8 & 15 & 0 & 1812.42 & 1707.92 & 0.06 & 1217.05 & 0.33 \\
\hline 14 & 14 & $\mathbf{0}$ & 20.86 & $\mathbf{5 0 . 8 7}$ & 1.44 & 107.26 & 4. 14 & 8 & 14 & 0 & 407.59 & 413.67 & 0.01 & 379.02 & 0.07 \\
\hline 14 & 13 & 0 & 499.89 & 478.94 & 0.04 & 460.92 & 0.08 & 8 & 13 & 0 & 385.09 & 366.66 & 0.05 & 287.63 & 0.25 \\
\hline 14 & 12 & 0 & 292.23 & 269.80 & 0.08 & 300.99 & 0.03 & 8 & 12 & 0 & 398.74 & 367.24 & 0.08 & 301.99 & 0.24 \\
\hline 14 & 11 & 0 & 606.21 & 580.16 & 0.04 & 525.56 & 0.13 & 8 & 11 & 0 & 722.81 & 683.22 & 0.05 & 518.26 & 0.28 \\
\hline 14 & 10 & 0 & 646.32 & 601.57 & 0.07 & 669.65 & 0.04 & 8 & 10 & 0 & 85.76 & 83.17 & 0.03 & 78.79 & 0.08 \\
\hline 14 & 9 & 0 & 860.07 & 820.86 & 0.05 & 787.53 & 0.08 & 8 & 9 & $\mathbf{0}$ & 8.01 & 27.15 & 2.39 & 39.13 & 3.89 \\
\hline 14 & 8 & 0 & 358.53 & 332.21 & 0.07 & 324.87 & 0.09 & 8 & 8 & 0 & 896.86 & 851.94 & 0.05 & 589.38 & 0.34 \\
\hline 14 & 7 & 0 & 681.36 & 668.68 & 0.02 & 576.98 & 0.15 & 8 & 7 & 0 & 556.19 & 524.18 & 0.06 & 419.52 & 0.25 \\
\hline 14 & 6 & 0 & 106.9 & 112.57 & 0.05 & 84.99 & 0.20 & 8 & 6 & 0 & 726.95 & 686.99 & 0.05 & 550.99 & 0.24 \\
\hline 14 & 5 & 0 & 126.26 & 132.99 & 0.05 & 90.37 & 0.28 & 8 & 5 & 0 & 801.94 & 759.75 & 0.05 & 588.94 & 0.27 \\
\hline 14 & 4 & 0 & 268.05 & 263.09 & 0.02 & 235.68 & 0.12 & 8 & 4 & 0 & 350.58 & 335.85 & 0.04 & 235.91 & 0.33 \\
\hline 13 & 15 & 0 & 485.27 & 462.25 & 0.05 & 405.44 & 0.16 & 7 & 15 & 0 & 218.99 & 234.03 & 0.07 & 173.69 & 0.21 \\
\hline 13 & 14 & 0 & 356.15 & 340.18 & 0.04 & 302.92 & 0.15 & 7 & 14 & 0 & 1709.09 & 1606.52 & 0.06 & 1708.67 & 0.00 \\
\hline 13 & 13 & 0 & 1149.61 & 1059.99 & 0.08 & 1160.89 & 0.01 & 7 & 13 & 0 & 1367.24 & 1285.66 & 0.06 & 1358.00 & 0.01 \\
\hline 13 & 12 & 0 & 216.53 & 214.50 & 0.01 & 174.26 & 0.20 & 7 & 12 & 0 & 662.9 & 625.29 & 0.06 & 624.81 & 0.06 \\
\hline 13 & 11 & 0 & 1223.02 & 1133.39 & 0.07 & 1196.68 & 0.02 & 7 & 11 & 0 & 1236.52 & 1145.95 & 0.07 & 1323.64 & 0.07 \\
\hline 13 & 10 & 0 & 426.65 & 403.27 & 0.05 & 358.40 & 0.16 & 7 & 10 & 0 & 311.26 & 289.61 & 0.07 & 333.50 & 0.07 \\
\hline 13 & 9 & 0 & 918.49 & 836.41 & 0.09 & 1002.50 & 0.09 & 7 & 9 & 0 & 378.71 & 356.56 & 0.06 & 351.76 & 0.07 \\
\hline 13 & 8 & 0 & 93.16 & 97.20 & 0.04 & 86.90 & 0.07 & 7 & 8 & 0 & 1498.04 & 1390.75 & 0.07 & 1427.53 & 0.05 \\
\hline 13 & 7 & 0 & 146.65 & 111.93 & 0.24 & 370.75 & 1.53 & 7 & 7 & 0 & 245.37 & 228.05 & 0.07 & 262.17 & 0.07 \\
\hline 13 & 6 & 0 & 893 & 824.27 & 0.08 & 855.68 & 0.04 & 7 & 6 & 0 & 671.73 & 629.14 & 0.06 & 662.50 & 0.01 \\
\hline 13 & 5 & 0 & 257.16 & 232.58 & 0.10 & 311.06 & 0.21 & 7 & 5 & 0 & 778.76 & 724.03 & 0.07 & 756.87 & 0.03 \\
\hline
\end{tabular}




\begin{tabular}{|c|c|c|c|c|c|c|c|c|c|c|c|c|c|c|c|}
\hline 13 & 4 & 0 & 1261.17 & 1171.24 & 0.07 & 1320.11 & 0.05 & 7 & 4 & 0 & 1146.02 & 1054.02 & 0.08 & 916.75 & 0.20 \\
\hline 12 & 15 & 0 & 52.75 & 57.86 & 0.10 & 49.83 & 0.06 & 6 & 15 & 0 & 940.32 & 863.88 & 0.08 & 1017.66 & 0.08 \\
\hline 12 & 14 & 0 & 848.74 & 797.85 & 0.06 & 738.27 & 0.13 & 6 & 14 & 0 & 420.59 & 401.57 & 0.05 & 337.22 & 0.20 \\
\hline 12 & 13 & 0 & 779.71 & 728.85 & 0.07 & 678.33 & 0.13 & 6 & 13 & 0 & 76.35 & 85.81 & 0.12 & 117.27 & 0.54 \\
\hline 12 & 12 & $\mathbf{0}$ & 71.84 & 99.87 & 0.39 & 175.15 & 1. 44 & 6 & 12 & 0 & 142.85 & 136.45 & 0.04 & 141.40 & 0.01 \\
\hline 12 & 11 & $\mathbf{0}$ & 33.66 & 54.62 & 0.62 & 110.16 & 2.27 & 6 & 11 & 0 & 259.31 & 246.29 & 0.05 & 292.42 & 0.13 \\
\hline 12 & 10 & 0 & 351.8 & 326.95 & 0.07 & 330.60 & 0.06 & 6 & 10 & 0 & 226.49 & 215.36 & 0.05 & 268.17 & 0.18 \\
\hline 12 & 9 & 0 & 179.03 & 170.95 & 0.05 & 143.44 & 0.20 & 6 & 9 & 0 & 1036.46 & 962.12 & 0.07 & 1047.75 & 0.01 \\
\hline 12 & 8 & 0 & 661.74 & 598.35 & 0.10 & 614.85 & 0.07 & 6 & 8 & 0 & 92.91 & 84.33 & 0.09 & 95.98 & 0.03 \\
\hline 12 & 7 & 0 & 1959.28 & 1801.15 & 0.08 & 2000.03 & 0.02 & 6 & 7 & 0 & 1658.77 & 1514.36 & 0.09 & 1541.82 & 0.07 \\
\hline 12 & 6 & 0 & 400.67 & 372.29 & 0.07 & 347.30 & 0.13 & 6 & 6 & 0 & 1572.69 & 1466.04 & 0.07 & 1701.61 & 0.08 \\
\hline 12 & 5 & 0 & 1451.56 & 1331.97 & 0.08 & 1401.46 & 0.03 & 6 & 5 & 0 & 2078.15 & 1949.36 & 0.06 & 2144.43 & 0.03 \\
\hline 12 & 4 & 0 & 117.12 & 104.85 & 0.10 & 136.19 & 0.16 & 6 & 4 & 0 & 1193.89 & 1118.89 & 0.06 & 1050.45 & 0.12 \\
\hline 11 & 15 & 0 & 1274.6 & 1177.02 & 0.08 & 1135.88 & 0.11 & 5 & 15 & 0 & 641.56 & 595.28 & 0.07 & 642.92 & 0.00 \\
\hline 11 & 14 & 0 & 1310.49 & 1197.69 & 0.09 & 1317.61 & 0.01 & 5 & 14 & 0 & 569.29 & 528.86 & 0.07 & 682.76 & 0.20 \\
\hline 11 & 13 & 0 & 155.39 & 141.02 & 0.09 & 147.16 & 0.05 & 5 & 13 & 0 & 641.29 & 595.14 & 0.07 & 669.67 & 0.04 \\
\hline 11 & 12 & 0 & 87.11 & 79.89 & 0.08 & 90.34 & 0.04 & 5 & 12 & 0 & 385.65 & 358.87 & 0.07 & 415.65 & 0.08 \\
\hline 11 & 11 & 0 & 601.96 & 551.54 & 0.08 & 587.95 & 0.02 & 5 & 11 & 0 & 227.69 & 214.54 & 0.06 & 204.94 & 0.10 \\
\hline 11 & 10 & 0 & 267.75 & 243.51 & 0.09 & 260.08 & 0.03 & 5 & 10 & 0 & 347.6 & 326.38 & 0.06 & 328.63 & 0.05 \\
\hline 11 & 9 & 0 & 345.26 & 309.20 & 0.10 & 362.41 & 0.05 & 5 & 9 & $\mathbf{0}$ & 20.71 & $\mathbf{5 7 . 5 3}$ & 1.78 & 114.41 & 4. 52 \\
\hline 11 & 8 & 0 & 1239.06 & 1137.83 & 0.08 & 1057.75 & 0.15 & 5 & 8 & 0 & 1539.322 & 1422.43 & 0.08 & 1380.10 & 0.10 \\
\hline 11 & 7 & 0 & 963.78 & 871.50 & 0.10 & 885.66 & 0.08 & 5 & 7 & 0 & 734.355 & 675.08 & 0.08 & 897.25 & 0.22 \\
\hline 11 & 6 & 0 & 169.48 & 172.22 & 0.02 & 179.56 & 0.06 & 5 & 6 & 0 & 1276.226 & 1172.88 & 0.08 & 1542.77 & 0.21 \\
\hline 11 & 5 & 0 & 4.7 & 75.16 & 14.99 & 169.74 & 35.1 & 5 & 5 & 0 & 1304.423 & 1220.63 & 0.06 & 1285.38 & 0.01 \\
\hline
\end{tabular}




\begin{tabular}{rrrrrrrr|rrrrrrrrr}
11 & 4 & 0 & 232.14 & 226.63 & 0.02 & 294.30 & 0.27 & 5 & 4 & 0 & 219.441 & 215.97 & 0.02 & 283.23 & 0.29 \\
10 & 15 & 0 & 1312.66 & 1217.34 & 0.07 & 1198.69 & 0.09 & 4 & 15 & 0 & 740.335 & 693.27 & 0.06 & 718.09 & 0.03 \\
10 & 14 & 0 & 523.55 & 476.59 & 0.09 & 555.52 & 0.06 & 4 & 14 & 0 & 696.137 & 654.89 & 0.06 & 785.23 & 0.13 \\
10 & 13 & 0 & 937.45 & 871.30 & 0.07 & 838.97 & 0.11 & 4 & 13 & 0 & 316.475 & 298.97 & 0.06 & 332.46 & 0.05 \\
10 & 12 & 0 & 27.12 & 26.18 & 0.03 & 44.19 & 0.63 & 4 & 12 & 0 & 248.249 & 238.62 & 0.04 & 183.95 & 0.26 \\
10 & 11 & 0 & 266.22 & 251.80 & 0.05 & 227.62 & 0.14 & 4 & 11 & 0 & 141.391 & 137.01 & 0.03 & 90.99 & 0.36 \\
10 & $\mathbf{1 0}$ & $\mathbf{0}$ & $\mathbf{4 4 . 0 3}$ & $\mathbf{9 6 . 2 3}$ & $\mathbf{1 . 1 9}$ & $\mathbf{1 7 6 . 7 2}$ & 3.01 & $\mathbf{4}$ & $\mathbf{1 0}$ & $\mathbf{0}$ & $\mathbf{9 . 4 7 3}$ & $\mathbf{1 2 . 5 6}$ & $\mathbf{0 . 3 3}$ & $\mathbf{2 7 . 5 3}$ & 1.91 \\
10 & 9 & 0 & 815.12 & 743.33 & 0.09 & 874.84 & 0.07 & 4 & 9 & 0 & 132.585 & 128.05 & 0.03 & 107.41 & 0.19 \\
10 & 8 & 0 & 257.22 & 260.94 & 0.01 & 257.20 & 0.00 & 4 & 8 & 0 & 611.249 & 579.59 & 0.05 & 503.28 & 0.18 \\
10 & 7 & 0 & 641.8 & 595.20 & 0.07 & 700.55 & 0.09 & 4 & 7 & 0 & 206.147 & 190.97 & 0.07 & 320.08 & 0.55 \\
10 & 6 & 0 & 248.37 & 233.29 & 0.06 & 265.64 & 0.07 & 4 & 6 & 0 & 437.267 & 410.74 & 0.06 & 591.85 & 0.35 \\
10 & 5 & 0 & 119.71 & 111.89 & 0.07 & 215.90 & 0.80 & 4 & 5 & 0 & 346.362 & 328.70 & 0.05 & 346.36 & 0.00 \\
10 & 4 & 0 & 617.62 & 576.90 & 0.07 & 650.90 & 0.05 & 4 & 4 & 0 & 1473.183 & 1384.90 & 0.06 & 1313.24 & 0.11 \\
\hline
\end{tabular}

